\title{
Non-Homogeneous Hydrodynamic Systems and Quasi-Stäckel Hamiltonians
}

\author{
Krzysztof MARCINIAK ${ }^{\dagger}$ and Maciej BEASZAK \\ $\dagger$ Department of Science and Technology, Campus Norrköping, Linköping University, Sweden \\ E-mail: krzma@itn.liu.se \\ $\ddagger$ Faculty of Physics, Division of Mathematical Physics, A. Mickiewicz University, \\ Poznań, Poland \\ E-mail: blaszakm@amu.edu.pl
}

Received June 12, 2017, in final form September 25, 2017; Published online September 28, 2017 https://doi.org/10.3842/SIGMA.2017.077

\begin{abstract}
In this paper we present a novel construction of non-homogeneous hydrodynamic equations from what we call quasi-Stäckel systems, that is non-commutatively integrable systems constructed from appropriate maximally superintegrable Stäckel systems. We describe the relations between Poisson algebras generated by quasi-Stäckel Hamiltonians and the corresponding Lie algebras of vector fields of non-homogeneous hydrodynamic systems. We also apply Stäckel transform to obtain new non-homogeneous equations of considered type.
\end{abstract}

Key words: Hamiltonian systems; superintegrable systems; Stäckel systems; hydrodynamic systems; Stäckel transform

2010 Mathematics Subject Classification: 70H06; 70H20; 35F50; 53B20

\section{Introduction}

In the present paper we investigate a class of first-order quasi-linear PDEs of the form

$$
q_{t}=K(q) q_{x}+Y(q),
$$

where $q=\left(q_{1}, \ldots, q_{n}\right)^{T}, q_{i}=q_{i}(x, t), K$ is an $n \times n$ matrix depending on $q$ and $Y$ is a vector depending on $q$ (vector field). The systems (1.1) are called in literature non-homogenous hydrodynamic or dispersionless systems. Specifically, we shall focus on the systems (1.1) with the homogenous part $K(q) q_{x}$ being semi-Hamiltonian in the sense of Tsarev [22] and weakly nonlinear [20]. The homogeneous case $Y=0$ has been intensively studied in literature due to the fact that such equations are strongly connected with classical integrable systems. For example, any solution of the homogeneous equation $q_{t}=K(q) q_{x}$ is also a solution of some particular Liouville integrable and separable finite-dimensional system, so called Stäckel system [10, 12]. The matrices $K(q)$ can in this context be interpreted as (1,1)-Killing tensors of Stäckel metrics [6].

The aim of this paper is to construct a class of non-homogeneous hydrodynamic equations of type (1.1) that can still be related to finite-dimensional integrable systems. This issue was first considered in $[11,13]$ but only for the case of two integrals, where the authors searched, for a given quadratic in momenta Hamiltonian, an integral of motion with quadratic in momenta terms but also having additional terms linear in momenta (magnetic terms), generating non-homogeneous terms in the related hydrodynamic equation. They noticed that these linear in momenta terms are given by vectors that are Killing vectors for the metric of the first Hamiltonian.

Another approach to the idea of constructing Liouville integrable systems with linear in momenta terms (restricted to cases $n=2$ and 3) has been presented in [18] and later in [17], where the authors defined these systems as so called quasi-Stäckel systems and also noted some connections of such systems with non-homogenous hydrodynamic equations. 
Inspired by these results we will look for a way - valid in any dimension - of modifying the Stäckel Hamiltonians by linear in momenta terms (which makes the corresponding hydrodynamic equations non-homogeneous) such that $(i)$ the modified system defines a maximally superintegrable system where the extra integrals of motion are all linear in momenta and (ii) it constitutes a non-commutative Poisson algebra. As a consequence, the structure constants of this algebra and the algebra of corresponding non-homogeneous hydrodynamic vector fields will be (up to a sign) the same. We call these modified systems quasi-Stäckel systems as well, but we want to stress that our definition of quasi-Stäckel systems is different from the definition in [17], as there the author demands these systems to be Liouville integrable while our systems are integrable only in the non-commutative sense.

The paper is organized as follows. In Section 2 we briefly remind the relation between homogeneous hydrodynamic Killing equations and geodesic Stäckel systems. In Section 3 we restrict our attention to a particular class of Stäckel systems, namely Benenti class with constant curvature metrics (it should be pointed out that there is a substantial literature on constant curvature systems with magnetic terms, see for example [16] and references there). Each system of this class consists of $n$ geodesic Hamiltonians $E_{i}$. For this particular class we find $n-1$ Killing vectors $Y_{i}$ of the metric tensor of the first Hamiltonian $E_{1}$, generating additional linear in momenta integrals $W_{i}$ which yields a maximally superintegrable Stäckel system. Then we investigate the Poisson algebras generated by the Hamiltonians $h_{i}=E_{i}+W_{i}$ which we call quasi-Stäckel Hamiltonians. Further, we show that the quasi-Stäckel Hamiltonians $h_{i}$ can be generated from a new type of linear relations that generalize the separation relations for the Stäckel Hamiltonians $E_{i}$. We call this new type of relations quasi-separation relations (which for $n=3$ are particular realizations of the quasi-Stäckel systems from [17]). In Section 4 we prove a theorem (Theorem 4.1) which establishes an explicit relation between Poisson algebras of $h_{i}$ and Lie-algebras of the corresponding non-homogeneous hydrodynamic vector fields (1.1). In Section 5 we exploit the notion of Stäckel transform [3, 4, 8, 14, 21] and analyze which of our systems $h_{i}$ can be mapped by this transform to new systems $\tilde{h}_{i}$ in such a way that the Hamiltonians $\tilde{h}_{i}$ also constitute an algebra. In this way we obtain new non-homogeneous hydrodynamic equations.

Let us point out that this article does not deal with the problem of integrability of the obtained non-homogeneous hydrodynamic equations. This is a separate problem yet to investigate.

\section{Homogeneous hydrodynamic Killing equations generated by geodesic Stäckel systems}

In this section we briefly remind some facts about the relation between hydrodynamic Killing equations and geodesic Stäckel systems.

Consider the following $n$ separation relations

$$
\sum_{j=1}^{n} \Phi_{i j}\left(\lambda_{i}\right) E_{j}=\frac{1}{2} f_{i}\left(\lambda_{i}\right) \mu_{i}^{2}, \quad i=1, \ldots, n,
$$

on $M=\mathbf{R}^{2 n}$, where $\Phi_{i j}$ and $f_{i}$ are arbitrary functions of one real variable. Since the $i$-th row of the matrix $\Phi(\lambda)$ depends on $\lambda_{i}$ only, it is the so called Stäckel matrix. The variables $(\lambda, \mu)=\left(\lambda_{1}, \ldots, \lambda_{n}, \mu_{1}, \ldots, \mu_{n}\right)$ will be in the sequel referred to as position and momentum separation coordinates on the phase space $M$. Solving the linear system (2.1) with respect to $E_{j}$ yields $n$ quadratic in momenta functions (Hamiltonians) on $M$

$$
E_{r}=\frac{1}{2} \mu^{T} A_{r} \mu, \quad r=1, \ldots, n,
$$


where $A_{r}$ are $n \times n$ matrices given by

$$
A_{r}=\operatorname{diag}\left(f_{1}\left(\lambda_{1}\right)\left(\Phi^{-1}\right)_{r 1}, \ldots, f_{n}\left(\lambda_{n}\right)\left(\Phi^{-1}\right)_{r n}\right), \quad r=1, \ldots, n .
$$

By their construction the functions $E_{r}$ are in involution with respect to the canonical Poisson bracket on $M$ given by $\left\{\lambda_{i}, \mu_{j}\right\}=\delta_{i j}$. They are referred to in literature as geodesic Stäckel Hamiltonians. Further, we can factorize $A_{r}$ as $A_{r}=K_{r} G$ with

$$
G=A_{1}=\operatorname{diag}\left(f_{1}\left(\lambda_{1}\right)\left(\Phi^{-1}\right)_{11}, \ldots, f_{n}\left(\lambda_{n}\right)\left(\Phi^{-1}\right)_{1 n}\right)
$$

and with

$$
K_{r}=\operatorname{diag}\left(\frac{\left(\Phi^{-1}\right)_{r 1}}{\left(\Phi^{-1}\right)_{11}}, \ldots, \frac{\left(\Phi^{-1}\right)_{r n}}{\left(\Phi^{-1}\right)_{1 n}}\right), \quad r=1, \ldots, n
$$

(so that $K_{1}=I$ ). From now on we will interpret the matrix $G$ as a contravariant form of a metric tensor on $M$. The corresponding covariant metric tensor will be denoted by $g$ so that $g G=I$. It can be shown that the matrices $K_{r}$ are then $(1,1)$-Killing tensors of the metric $G$. For a fixed Stäckel matrix $\Phi$ we have thus the whole family of metrics $G$ parametrized by $n$ arbitrary functions $f_{i}$ of one variable. The tensors $K_{r}$ are then Killing tensors for any metric from this family. Thus, the Stäckel Hamiltonians $E_{r}$ are geodesic Hamiltonians of a Liouville integrable system in the Riemannian space $(M, g)$. The Hamiltonian equations for $E_{r}$ are given by

$$
\lambda_{t_{r}}=\frac{\partial E_{r}}{\partial \mu}, \quad \mu_{t_{r}}=-\frac{\partial E_{r}}{\partial \lambda}, \quad r=1, \ldots, n,
$$

with the implicit common solution for all the positions $\lambda_{i}=\lambda_{i}\left(t_{1}, \ldots, t_{n}\right)$ in the form

$$
\sum_{k=1}^{n} \int^{\lambda_{k}} \frac{\Phi_{k, n-r}(\xi)}{\varphi_{k}(\xi)} d \xi=t_{r}, \quad r=1, \ldots, n,
$$

where

$$
\varphi_{k}(\xi)=\left(\frac{1}{2} f_{k}(\xi) \sum_{j=1}^{n} \Phi_{k j}(\xi) a_{j}\right)^{\frac{1}{2}}
$$

and $a_{j}$ are arbitrary constants parametrizing the Liouville tori (values of Hamiltonians $E_{r}$ ). The first part of Hamiltonian equations (2.4) can be explicitly written as $\lambda_{t_{r}}=K_{r} G \mu$ so that $G \mu=\lambda_{t_{1}}$ and every solution $\lambda_{i}\left(t_{1}, \ldots, t_{n}\right)$ of (2.4) satisfies the following system of hydrodynamic equations

$$
\lambda_{t_{r}}=K_{r}(\lambda) \lambda_{x} \equiv Z_{r}\left(\lambda, \lambda_{x}\right), \quad r=2, \ldots, n,
$$

where we denoted $t_{1}=x$. We call the system (2.7) a hydrodynamic Killing system. The differential functions $Z_{r}$ can be interpreted as vector fields on an infinite-dimensional space of functions $\lambda(x)$ and since $\left\{E_{i}, E_{j}\right\}=0$ one can show that also the vector fields $Z_{r}$ commute: $\left[Z_{r}, Z_{s}\right]=0$ for all $r, s=1, \ldots, n$ (where $Z_{1}=\lambda_{x}$ is the translational symmetry). The system (2.7) is also known in the literature as a weakly nonlinear semi-Hamilton system and since $K_{r}$ are diagonal in $\lambda$-variables the variables $\lambda$ are in this context known as Riemann invariants. The general solution of (2.7) is also given by (2.5) if we allow $\varphi_{k}$ to be arbitrary. Therefore, due to (2.6), any solution of the hydrodynamic system (2.7) can be obtained as a solution of a particular Stäckel system with the suitably chosen functions $f_{i}$. 


\section{Quasi-Stäckel systems}

The aim of this paper is to construct non-homogeneous hydrodynamic equations that can still be related to some finite-dimensional integrable systems. The first steps in that direction was made by Ferapontov and Fordy in $[11,13]$. As we wrote in Introduction we will modify the geodesic Hamiltonians $E_{i}$ by linear in momenta constants of motion $W_{i}$ for Hamiltonian $E_{1}$, which makes the corresponding hydrodynamic equations non-homogeneous, so that $(i)$ the modified system defines a maximally superintegrable system where the extra integrals of motion are all linear in momenta and $(i i)$ it constitutes a non-commutative Poisson algebra. As a consequence, the structure constants of this algebra and the algebra of corresponding hydrodynamic vector fields will be (up to a sign) the same.

It is well known that the linear in momenta function $W=p^{T} Y=\sum_{i} p_{i} Y^{i}$ on $M$ is an integral of motion of $E_{1}$ if and only if $Y=\sum_{i} Y^{i}(q) \frac{\partial}{\partial q_{i}}$ is a Killing vector for the metric $G$. Therefore, we have to find enough Killing vectors $Y_{r}$ of $G$ in order to perform our task. In analogy with the homogeneous case, the first part of Hamiltonian equations (2.4) for the modified Hamiltonians $h_{r}=E_{r}+W_{r}$ will then take the non-homogenous form

$$
\lambda_{t_{r}}=K_{r}(\lambda) \lambda_{x}+Y_{r}(\lambda) \equiv Z_{r}\left(\lambda, \lambda_{x}\right), \quad r=2, \ldots, n,
$$

where $W_{r}=p^{T} Y_{r}$.

We will solely work with the metrics of constant curvature. It is well known that the Lie algebra of Killing vectors of constant curvature metrics is of the maximal dimension $n(n+1) / 2$, but the problem of identifying all the constant-curvature metrics of the form (2.3) is not solved yet, so we will focus on some particular classes of metrics of the form (2.3) which are known to be of constant curvature.

\subsection{Benenti class of Stäckel systems}

We will thus impose two restrictions on the metric $G$. Firstly, we will only consider a class of Stäckel systems (2.1) given by the Stäckel matrix of the very particular form $\Phi_{i j}=\lambda_{i}^{n-j}$. This results in the following separation relations

$$
\sum_{j=1}^{n} \lambda_{i}^{n-j} E_{j}=\frac{1}{2} f_{i}\left(\lambda_{i}\right) \mu_{i}^{2}, \quad i=1, \ldots, n .
$$

Such systems are called in literature Benenti systems. Moreover, in this case the metric tensor (2.3) attains the explicit form

$$
G=A_{1}=\operatorname{diag}\left(\frac{f_{1}\left(\lambda_{1}\right)}{\Delta_{1}}, \ldots, \frac{f_{n}\left(\lambda_{n}\right)}{\Delta_{n}}\right), \quad \Delta_{i}=\prod_{j \neq i}^{n}\left(\lambda_{i}-\lambda_{j}\right),
$$

while the Killing tensors $K_{r}$ are

$$
K_{r}=(-1)^{r+1} \operatorname{diag}\left(\frac{\partial \sigma_{r}}{\partial \lambda_{1}}, \ldots, \frac{\partial \sigma_{r}}{\partial \lambda_{n}}\right), \quad r=1, \ldots, n
$$

where $\sigma_{r}(\lambda)$ are elementary symmetric polynomials in $\lambda$.

Secondly, we will also assume that all $f_{i}$ are equal to the same monomial of order not exceeding $n+1$

$$
f_{i}\left(\lambda_{i}\right)=\lambda_{i}^{m}, \quad m \in\{0, \ldots, n+1\},
$$


which renders $n+2$ metrics

$$
G_{m}=\operatorname{diag}\left(\frac{\lambda_{1}^{m}}{\Delta_{1}}, \ldots, \frac{\lambda_{n}^{m}}{\Delta_{n}}\right), \quad m \in\{0, \ldots, n+1\}
$$

with the corresponding geodesic Stäckel Hamiltonians

$$
E_{r}^{m}=\frac{1}{2} \mu^{T} K_{r} G_{m} \mu, \quad r=1, \ldots, n
$$

Remark 3.1. It can be shown that the metric $G_{m}$ is flat for $m \in\{0, \ldots, n\}$ and of constant curvature for $m=n+1$. The separation variables $\lambda_{i}$ can in this case be considered as appropriate degenerations of Jacobi elliptic coordinates, see [15].

In the separation coordinates (Riemann invariants) $\lambda$ the hydrodynamic equations (2.7) take a particular form

$$
\frac{d \lambda}{d t_{r}}=(-1)^{r+1} \frac{\partial \sigma_{r}}{\partial \lambda} \lambda_{x}, \quad r=1, \ldots, n
$$

It turns out that the search for the linear in momenta integrals for $E_{1}$ is much easier in the coordinates

$$
q_{i}(\lambda)=(-1)^{i} \sigma_{i}(\lambda)
$$

We will refer to them as Viète coordinates. The corresponding conjugate momenta $p(\lambda, \mu)$ on $M$ are given by

$$
p_{i}=-\sum_{k=1}^{n} \frac{\lambda_{k}^{n-i}}{\Delta_{k}} \mu_{k}, \quad i=1, \ldots, n
$$

In $q$-variables the components of $G_{m}$ are polynomials in $q$ given by [5]

$$
\begin{aligned}
& \left(G_{0}\right)^{i j}=\sum_{k=0}^{n-1} q^{k} \delta_{n+k+1}^{i+j}, \\
& \left(G_{m}\right)^{i j}= \begin{cases}\sum_{k=0}^{n-m-1} q_{k} \delta_{n-m+k+1}^{i+j}, \quad i, j=1, \ldots, n-m, \\
-\sum_{k=n-m+1}^{n} q_{k} \delta_{n-m+k+1}^{i+j}, & i, j=n-m+1, \ldots, n, \quad m=1, \ldots, n, \\
0, & \text { otherwise, }\end{cases} \\
& \left(G_{n+1}\right)^{i j}=q_{i} q_{j}-q_{i+j}, \quad i, j=1, \ldots, n
\end{aligned}
$$

(where we denote $q_{0}=1$ ), while the hydrodynamic equations (2.7) attain the form $q_{t_{r}}=K_{r}(q) q_{x}$ or, explicitly [2]

$$
\frac{d q_{j}}{d t_{r}}=\left(Z_{r}\right)^{j}=\left(q_{j+r-1}\right)_{x}+\sum_{k=1}^{j-1}\left(q_{k}\left(q_{j+r-k-1}\right)_{x}-q_{j+r-k-1}\left(q_{k}\right)_{x}\right), \quad r, j=1, \ldots, n
$$




\subsection{Killing vectors and additional integrals of motion}

Let us define the following sets of indices:

$$
I_{1}^{m}=\{2, \ldots, n-m+1\}, \quad I_{2}^{m}=\{n-m+2, \ldots, n\}, \quad m=0, \ldots, n+1 .
$$

The search for linear in momenta extra integrals of motion for $E_{1}^{m}$ that are also linear in $q$ yields the following lemma.

Lemma 3.2. For $m=0, \ldots, n-1$ the functions

$$
W_{r}^{m}=\sum_{i=1}^{r-1} i q_{r-i-1} p_{n-m-i+1}, \quad r \in I_{1}^{m},
$$

and for $m=2, \ldots, n+1$ the functions

$$
W_{r}^{m}=\sum_{i=1}^{n-r+1} i q_{r+i-1} p_{n-m+i+1}, \quad r \in I_{2}^{m}
$$

are constants of motion for $E_{1}^{m}$.

For each $m$ between 1 and $n$, this lemma yields $n-1$ (and $n$ in case $m=0$ and $m=n+1$ ) linear in both momenta $p$ and positions $q$ additional constants of motion for $E_{1}^{m}$ with the corresponding Killing vector fields $Y_{r}^{m}$ for $G_{m}$ so that

$$
W_{r}^{m}=p^{T} Y_{r}^{m}=\sum_{i=1}^{n} p_{i}\left(Y_{r}^{m}\right)^{i}
$$

Lemma 3.3. For each $m \in\{0, \ldots, n+1\}$ the $2 n-1$ functions

$$
E_{1}^{m}, \ldots, E_{n}^{m}, W_{2}^{m}, \ldots, W_{n}^{m}
$$

constitute a maximally superintegrable system with respect to $E_{1}^{m}$.

Proof. It is well known that if a Stäckel metric $G(q)$ on $Q \subset R^{n}$ is of constant curvature then there exists $n-1$ independent second-order contravariant Killing tensors $A_{2}(q), \ldots, A_{n}(q)$ of metric $G$, which commute with respect to the Schouten bracket (4.6): $\left[A_{i}, A_{j}\right]_{S}=0, i, j=$ $2, \ldots, n$ and there exists $\frac{1}{2} n(n+1)$ Killing vectors $Y_{i}(q)$ of $G$. In consequence, on the phase space $T^{*} Q$, there exist $n$ functions quadratic in momenta $E_{1}=\frac{1}{2} p^{T} G(q) p, E_{2}=\frac{1}{2} p^{T} A_{2}(q) p, \ldots, E_{n}=$ $\frac{1}{2} p^{T} A_{n}(q) p$ in involution with respect to the canonical Poisson bi-vector and $\frac{1}{2} n(n+1)$ linear in momenta functions $W_{i}=p^{T} Y_{i}(q)$, forming a Poisson algebra. In the set $(E, W)$ of $n+n(n+1) / 2$ functions there exist two types of functional relations: 1) all $E_{i}$ are expressible by $W$-functions, i.e., $E_{i}=E_{i}(W), i=1, \ldots, n$ and $2 \varphi_{k}(W)=0$, where $\varphi_{k}$ is an appropriate number (depending on $n$ ) of functions, quadratic in all $W$. Now, in order to prove the functional independence of the subset (3.12), it is sufficient to show than none of these relations survive in the set (3.12). The functional independence of $W_{2}, \ldots, W_{n}$ follows from the fact that the matrix of differentials $d W_{2}^{m}, \ldots, d W_{n}^{m}$ has for each $m$ the maximal rank $n-1$. Further, since for each $m$ all $W_{i}^{m}$, $i=2, \ldots, n$ contain together less number of $q^{\prime} s$ then any $E_{j}$ does, so $E_{j} \neq E_{j}\left(W_{2}, \ldots, W_{n}\right)$, $j=1, \ldots, n$.

From (3.10), (3.11) and (3.6), (3.7) it follows that in the Riemann invariants $\lambda$ the corresponding Killing vectors $Y_{r}^{m}$ of metrics $G_{m}$ are represented by the formulas

$$
\left(Y_{r}^{m}\right)^{i}=\sum_{k=1}^{r-1}(-1)^{r-k} k \sigma_{r-k-1} \frac{\lambda_{i}^{m+k-1}}{\Delta_{i}}, \quad r \in I_{1}^{m}
$$


and

$$
\left(Y_{r}^{m}\right)^{i}=\sum_{k=1}^{n-r+1}(-1)^{r+k} k \sigma_{r+k-1} \frac{\lambda_{i}^{m-k-1}}{\Delta_{i}}, \quad r \in I_{2}^{m}
$$

An important observation is that both $E_{r}^{m}$ and $W_{r}^{m}$ are homogeneous functions of the same order $n-m-r+2$ with respect to the scaling

$$
p_{k} \rightarrow \varepsilon^{k} p_{k}, \quad q_{k} \rightarrow \varepsilon^{-k} q_{k},
$$

so it is natural to consider their sums when constructing Hamiltonians with linear in momenta terms. Let us thus define, for each $m$, the $n$ functions

$$
h_{1}^{m}=E_{1}^{m}, \quad h_{r}^{m}=E_{r}^{m}+W_{r}^{m}, \quad r=2, \ldots, n .
$$

As we mentioned in introduction, we call the functions $h_{r}^{m}$ quasi-Stäckel Hamiltonians.

Theorem 3.4. The functions $h_{r}^{m}$, for each fixed $m$, constitute a Poisson algebra $\mathfrak{g}=\operatorname{Span}\left\{h_{r}^{m}\right.$ : $r=1, \ldots, n\}$ with the following commutation relations for $i, j=2, \ldots, n$ :

$$
\left\{h_{i}^{m}, h_{j}^{m}\right\}= \begin{cases}0, & \text { for } i \in I_{1}^{m} \text { and } j \in I_{2}^{m}, \\ (j-i) h_{i+j-(n-m+2)}^{m}, & \text { for } i, j \in I_{1}^{m}, \\ -(j-i) h_{i+j-(n-m+2)}^{m}, & \text { for } i, j \in I_{2}^{m}\end{cases}
$$

where we use the notation $h_{i}^{m}=0$ for $i \leq 0$ or $i>n$.

Note that for the cases $n=2$ and $n=3$ this algebra is commutative.

Proof. We have

$$
\left\{h_{i}^{m}, h_{j}^{m}\right\}=\left\{E_{i}^{m}, E_{j}^{m}\right\}+\left\{E_{i}^{m}, W_{j}^{m}\right\}+\left\{W_{i}^{m}, E_{j}^{m}\right\}+\left\{W_{i}^{m}, W_{j}^{m}\right\},
$$

where the first term is zero as the Stäckel Hamiltonians $E_{i}^{m}$ pairwise commute. By a direct calculation one can show that the functions $W_{i}^{m}$ themselves constitute a Poisson algebra with the same structure constants as in (3.16). Finally, a calculation involving the formulas (3.8) and (3.9) shows that for $i, j=2, \ldots, n$

$$
\left\{E_{i}^{m}, W_{j}^{m}\right\}+\left\{W_{i}^{m}, E_{j}^{m}\right\}= \begin{cases}0, & \text { for } i \in I_{1}^{m} \text { and } j \in I_{2}^{m} \\ (j-i) E_{i+j-(n-m+2)}^{m}, & \text { for } i, j \in I_{1}^{m}, \\ -(j-i) E_{i+j-(n-m+2)}^{m}, & \text { for } i, j \in I_{2}^{m} .\end{cases}
$$

In consequence, (3.17) yields the right-hand side of (3.16).

Due to the form of (3.16) the algebra $\mathfrak{g}$ splits into a direct sum of two algebras $\mathfrak{g}=\mathfrak{g}_{1} \oplus \mathfrak{g}_{2}$ where $\mathfrak{g}_{1}=\operatorname{Span}\left\{h_{i}: i \in I_{1}^{m}\right\}$ and $\mathfrak{g}_{2}=\operatorname{Span}\left\{h_{i}: i \in I_{2}^{m}\right\}$.

Remark 3.5. The functions $h_{1}^{m}, \ldots, h_{n}^{m}, W_{2}^{m}, \ldots, W_{n}^{m}$ constitute a non-commutative integrable system $[7,19]$. 


\subsection{Quasi-separation relations}

Let us consider the following linear relations (cf. (2.1))

$$
\sum_{j=1}^{n} \Phi_{i j}\left(\lambda_{i}\right) h_{j}=\frac{1}{2} f_{i}\left(\lambda_{i}\right) \mu_{i}^{2}+\sum_{k=1}^{n} u_{i k}(\lambda) \mu_{k}, \quad i=1, \ldots, n,
$$

with a given Stäckel matrix $\Phi$, with $n$ arbitrary functions $f_{i}$ of one variable and with a set of $n^{2}$ functions $u_{i k}(\lambda)$ depending in general on all $\lambda_{j}$. Similar relations were previously studied in $[17,18]$. Solving $(3.19)$ with respect to $h_{i}$ we obtain

$$
h_{r}=E_{r}+W_{r}, \quad r=1, \ldots, n,
$$

such that $E_{r}$ are the geodesic Stäckel Hamiltonians (2.2), so they are in mutual involution, and $W_{r}=p^{T} Y_{r}$ are some linear in momenta terms (magnetic terms).

Assuming, additionally, that $(i) W_{1}=0$ and $(i i)\left\{h_{1}, h_{j}\right\}=0$ for $j=2, \ldots, n$ we obtain that $Y_{r}$ are Killing vectors of the metric $G$ in $E_{1}$. Thus, we obtain that $E_{1}, \ldots, E_{n}, W_{2}, \ldots, W_{n}$ constitute a maximally superintegrable and separable system. In such a case the relations (3.19) will be called quasi-separation relations.

Theorem 3.6. The maximally superintegrable system (3.12) is generated by the following quasiseparation relations:

$$
\sum_{j=1}^{n} \lambda_{i}^{n-j} h_{j}=\frac{1}{2} \lambda_{i}^{m} \mu_{i}^{2}+\sum_{k=1}^{n} u_{i k}(\lambda) \mu_{k}, \quad i=1, \ldots, n,
$$

where

$$
\sum_{k=1}^{n} u_{i k}(\lambda) \mu_{k}= \begin{cases}-\sum_{k \neq i} \frac{\mu_{i}-\mu_{k}}{\lambda_{i}-\lambda_{k}}, & \text { for } m=0, \\ -\lambda_{i}^{m-1} \sum_{k \neq i} \frac{\lambda_{i} \mu_{i}-\lambda_{k} \mu_{k}}{\lambda_{i}-\lambda_{k}}+(m-1) \lambda_{i}^{m-1} \mu_{i}, & \text { for } m=1, \ldots, n, \\ -\lambda_{i}^{n-1} \sum_{k \neq i} \frac{\lambda_{i}^{2} \mu_{i}-\lambda_{k}^{2} \mu_{k}}{\lambda_{i}-\lambda_{k}}+(n-1) \lambda_{i}^{n} \mu_{i}, & \text { for } m=n+1 .\end{cases}
$$

Thus, by solving (3.20), (3.21) with respect to $h_{j}$ we obtain all the Hamiltonians (3.4) as well as the additional constants $W_{i}^{m}(3.10),(3.11)$ of the Hamiltonian $E_{1}^{m}$.

\section{Non-homogeneous hydrodynamic equations of Killing type}

In this section we prove a theorem describing the relation between the systems presented in Section 3 and non-homogeneous hydrodynamic equations. Consider the set of Hamiltonians on $\mathbf{R}^{2 n}$

$$
h_{r}=E_{r}+W_{r}, \quad E_{r}=\frac{1}{2} p^{T} A_{r} p, \quad W_{r}=p^{T} Y_{r}, \quad r=1, \ldots, n,
$$

with

$$
\left\{E_{r}, E_{s}\right\}=0, \quad r, s=1, \ldots, n,
$$


where $A_{r}=K_{r} G$ with $L_{Y_{r}} G=0$ (i.e., all $Y_{r}$ are Killing vectors for the metric $G$ ). Suppose also that the functions $W_{r}$ constitute a non-commutative Poisson algebra with some structure constants $c_{r s}^{i}$

$$
\left\{W_{r}, W_{s}\right\}=\sum_{i=1}^{n} c_{r s}^{i} W_{i}
$$

and moreover that the following condition holds

$$
\left\{E_{r}, W_{s}\right\}+\left\{W_{r}, E_{s}\right\}=\sum_{i=1}^{n} c_{r s}^{i} E_{i} .
$$

The conditions (4.2) and (4.3) imply that $h_{r}$ also constitute a non-commutative Poisson algebra with the same structure constants $c_{r s}^{i}$

$$
\left\{h_{r}, h_{s}\right\}=\sum_{i=1}^{n} c_{r s}^{i} h_{i}
$$

In order to relate the algebra (4.4) with an appropriate algebra of hydrodynamic vector fields we will use the link between the canonical Poisson bracket and the Schouten bracket between symmetric contravariant tensors. Actually, for a pair of functions on $M$

$$
F_{K}=\frac{1}{k !} K^{i_{1} \ldots i_{k}}(q) p_{i_{1}} \cdots p_{i_{k}}, \quad F_{R}=\frac{1}{r !} R^{i_{1} \ldots i_{r}}(q) p_{i_{1}} \cdots p_{i_{r}}
$$

(we use for the moment the Einstein summation convention) the following relation holds [9]

$$
\left\{F_{K}, F_{R}\right\}=-\left([K, R]_{S}\right)^{i_{1} \ldots i_{k+r-1}} p_{i_{1}} \cdots p_{i_{k+r-1}}
$$

where

$$
\left([K, R]_{S}\right)^{l_{1} \ldots l_{k+r-1}}=\frac{1}{k ! r !}\left[k K^{i\left(l_{1} \ldots\right.} \partial_{n} R^{\left.\cdots l_{k+r-1}\right)}-r R^{i\left(l_{1} \ldots\right.} \partial_{i} K^{\left.\cdots l_{k+r-1}\right)}\right], \quad \partial_{i}=\frac{\partial}{\partial q_{i}}
$$

defines the Schouten bracket on $\mathbf{R}^{n}$ (the configuration space) and where (...) is the symmetrization operation over the indices.

Formula (4.5) implies that the vector fields $Y_{r}$ constitute a non-abelian Lie algebra with the structure constants $-c_{r s}^{i}$

$$
\left[Y_{r}, Y_{s}\right]=-\sum_{i=1}^{n} c_{r s}^{i} Y_{i}
$$

and that

$$
\left[A_{r}, Y_{s}\right]_{S}+\left[Y_{r}, A_{s}\right]_{S}=-\sum_{i=1}^{n} c_{r s}^{i} A_{i}
$$

From the properties of the Schouten bracket it follows that $\left[Y_{r}, A_{s}\right]_{S}=L_{Y_{r}} A_{s}$. Since moreover $L_{Y_{s}} A_{r}=\left(L_{Y_{s}} K_{r}\right) G$, as $Y_{s}$ are Killing vectors of a non-singular metric $G$, we obtain from (4.8)

$$
L_{Y_{r}} K_{s}-L_{Y_{s}} K_{r}=-\sum_{i=1}^{n} c_{r s}^{i} K_{i} .
$$


Theorem 4.1. Consider the non-homogeneous hydrodynamic systems

$$
q_{t_{r}}=K_{r} q_{x}+Y_{r} \equiv Z_{r} \quad r=2, \ldots, n
$$

where $K_{r}$ and $Y_{r}$ are such that the conditions (4.1)-(4.3) are satisfied. Then

$$
\left[Z_{r}, Z_{s}\right]=-\sum_{i=1}^{n} c_{r s}^{i} Z_{i}
$$

Proof. We have

$$
\left[Z_{r}, Z_{s}\right]=\left[K_{r} q_{x}+Y_{r}, K_{s} q_{x}+Y_{s}\right]=\left[K_{r} q_{x}, K_{s} q_{x}\right]+\left[K_{r} q_{x}, Y_{s}\right]+\left[Y_{r}, K_{s} q_{x}\right]+\left[Y_{r}, Y_{s}\right] .
$$

To begin with, $\left[K_{r} q_{x}, K_{s} q_{x}\right]=0$ for all $r, s$ (see for example [12]). Further, as $\left[Y_{s}, q_{x}\right]=0$ we have $\left[Y_{s}, K_{r} q_{x}\right]=\left(L_{Y_{s}} K_{r}\right) q_{x}$. Using (4.7) and (4.9) we obtain

$$
\left[Z_{r}, Z_{s}\right]=\left(L_{Y_{r}} K_{s}-L_{Y_{s}} K_{r}\right) q_{x}-\sum_{i=1}^{n} c_{r s}^{i} Y_{i}=-\sum_{i=1}^{n} c_{r s}^{i}\left(K_{i} q_{x}+Y_{i}\right)=-\sum_{i=1}^{n} c_{r s}^{i} Z_{i} .
$$

Thus, the vector fields $Z_{r}$ in (4.10) constitute a Lie algebra with up to a sign the same structure constants as the Poisson algebra (4.4). Besides, since our systems (3.15) with structure constants (3.16) satisfy the conditions (4.1)-(4.3) (as (3.18) is a specification of (4.3)) we see that they possess non-homogeneous hydrodynamic counterparts, with the same structure constants.

Example 4.2. Let us consider the case $n=4$ in Viète coordinates $q$. As $m=0, \ldots, n+1$ we have then $n+2=6$ different non-homogeneous hydrodynamic systems (4.10):

$$
\begin{aligned}
& {\left[\begin{array}{l}
q_{1} \\
q_{2} \\
q_{3} \\
q_{4}
\end{array}\right]_{t_{2}}=\left[\begin{array}{cccc}
0 & 1 & 0 & 0 \\
-q_{2} & q_{1} & 1 & 0 \\
-q_{3} & 0 & q_{1} & 1 \\
-q_{4} & 0 & 0 & q_{1}
\end{array}\right]\left[\begin{array}{l}
q_{1} \\
q_{2} \\
q_{3} \\
q_{4}
\end{array}\right]_{x}+Y_{2} \equiv Z_{2},} \\
& {\left[\begin{array}{l}
q_{1} \\
q_{2} \\
q_{3} \\
q_{4}
\end{array}\right]_{t_{3}}=\left[\begin{array}{cccc}
0 & 0 & 1 & 0 \\
-q_{3} & 0 & q_{1} & 1 \\
-q_{4} & -q_{3} & q_{2} & q_{1} \\
-0 & -q_{4} & 0 & q_{2}
\end{array}\right]\left[\begin{array}{l}
q_{1} \\
q_{2} \\
q_{3} \\
q_{4}
\end{array}\right]_{x}+Y_{3} \equiv Z_{3},} \\
& {\left[\begin{array}{l}
q_{1} \\
q_{2} \\
q_{3} \\
q_{4}
\end{array}\right]_{t_{4}}=\left[\begin{array}{cccc}
0 & 0 & 0 & 1 \\
-q_{4} & 0 & 0 & q_{1} \\
0 & -q_{4} & 0 & q_{2} \\
0 & 0 & -q_{4} & q_{3}
\end{array}\right]\left[\begin{array}{l}
q_{1} \\
q_{2} \\
q_{3} \\
q_{4}
\end{array}\right]_{x}+Y_{4} \equiv Z_{4},}
\end{aligned}
$$

where

$$
\begin{array}{llll}
\text { for } m=0: & Y_{2}=(0,0,0,1)^{T}, & Y_{3}=\left(0,0,2, q_{1}\right)^{T}, & Y_{4}=\left(0,3,2 q_{1}, q_{2}\right)^{T}, \\
\text { for } m=1: & Y_{2}=(0,0,1,0)^{T}, & Y_{3}=\left(0,2, q_{1}, 0\right)^{T}, & Y_{4}=\left(3,2 q_{1}, q_{2}, 0\right)^{T}, \\
\text { for } m=2: & Y_{2}=(0,1,0,0)^{T}, & Y_{3}=\left(2, q_{1}, 0,0\right)^{T}, & Y_{4}=\left(0,0,0, q_{4}\right)^{T}, \\
\text { for } m=3: & Y_{2}=(1,0,0,0)^{T}, & Y_{3}=\left(0,0, q_{3}, 2 q_{4}\right), & Y_{4}=\left(0,0, q_{4}, 0\right)^{T}, \\
\text { for } m=4: & Y_{2}=\left(0, q_{2}, 2 q_{3}, 3 q_{4}\right)^{T}, & Y_{3}=\left(0, q_{3}, 2 q_{4}, 0\right)^{T}, & Y_{4}=\left(0, q_{4}, 0,0\right)^{T}, \\
\text { for } m=5: & Y_{2}=\left(q_{2}, 2 q_{3}, 3 q_{4}, 0\right)^{T}, & Y_{3}=\left(q_{3}, 2 q_{4}, 0,0\right)^{T}, & Y_{4}=\left(q_{4}, 0,0,0\right)^{T},
\end{array}
$$

which constitute appropriate Lie algebras with the following nonzero elements given by Theorem 4.1 and (3.16)

$$
\text { for } m=0: \quad\left[Z_{3}, Z_{4}\right]=-Z_{1},
$$




$$
\begin{array}{lll}
\text { for } m=1: & {\left[Z_{2}, Z_{4}\right]=-2 Z_{1}, \quad\left[Z_{3}, Z_{4}\right]=-Z_{2},} \\
\text { for } m=2: & {\left[Z_{2}, Z_{3}\right]=-Z_{1},} & \\
\text { for } m=3: & {\left[Z_{3}, Z_{4}\right]=Z_{4},} \\
\text { for } m=4: & {\left[Z_{2}, Z_{3}\right]=Z_{3}, \quad\left[Z_{2}, Z_{4}\right]=2 Z_{4},} \\
\text { for } m=5: & {\left[Z_{2}, Z_{3}\right]=Z_{3},}
\end{array}
$$

where $Z_{1}=\left(q_{1}, q_{2}, q_{3}, q_{4}\right)_{x}^{T}$. Using (3.5), (3.13) and (3.14) the present example can be easily calculated in the Riemman invariants $\lambda$.

\section{Stäckel transform and new non-homogeneous hydrodynamic Killing systems}

Stäckel transform is a functional transform that maps a Liouville integrable system into a new integrable system, and in particular it maps a Stäckel system into a new Stäckel systems [3, 8, 14, 21], which explains its name. In [4] the authors considered the action of Stäckel transform on superintegrable systems in such a way that it preserves superintegrability. It was found that only particular one-parameter Stäckel transforms preserve superintegrability. Here we demonstrate that Stäckel transforms are also applicable for our particular systems (3.15) defined by the quasi-separation relations (3.20). Nevertheless, if we demand that the transformed system shall also constitute a Poisson algebra, then the number of admissible Stäckel transforms becomes very limited.

Consider thus Hamiltonians (3.15) extended by some potentials $V_{r}^{(k)}$

$$
\begin{aligned}
& h_{1}^{m}=E_{1}^{m}+\alpha V_{1}^{(k)} \equiv H_{1}^{m}, \\
& h_{r}^{m}=E_{r}^{m}+\alpha V_{r}^{(k)}+W_{r}^{m} \equiv H_{r}^{m}+W_{r}^{m}, \quad r=2, \ldots, n,
\end{aligned}
$$

where $\alpha$ is a parameter, and where the potentials $V_{r}^{(k)}, k \in \mathbf{Z}$, are defined by the following separation relations

$$
\lambda_{i}^{k}+\sum_{j=1}^{n} \lambda_{i}^{n-j} V_{j}^{(k)}=0, \quad i=1, \ldots, n .
$$

As such, the potentials $V_{r}^{(k)}$ are called basic separable potentials. Thus, the functions $h_{r}^{m}$ in (5.1) are generated by the following quasi-separation relations

$$
\alpha \lambda_{i}^{k}+\sum_{j=1}^{n} \lambda_{i}^{n-j} h_{j}=\frac{1}{2} \lambda_{i}^{m} \mu_{i}^{2}+\sum_{k=1}^{n} u_{i k}(\lambda) \mu_{k}, \quad i=1, \ldots, n,
$$

with $u_{i k}$ as in (3.21), i.e., the term $\lambda_{i}^{k}$ in (5.3) generates the potential $V_{r}^{(k)}$ in (5.1).

Lemma 5.1. The functions $H_{1}^{m}, \ldots, H_{n}^{m}, W_{2}^{m}, \ldots, W_{n}^{m}$ constitute a maximally superintegrable (with respect to $\left.H_{1}^{m}\right)$ system only in the following four cases: $(m, k)=(0, n),(n, n),(1,-1)$ and $(n+1,-1)$.

Proof. Since the functions $H_{r}^{m}$ are Stäckel Hamiltonians (albeit no longer geodesic), they commute: $\left\{H_{r}^{m}, H_{s}^{m}\right\}=0$ for any $r, s$, so $H_{1}^{m}, \ldots, H_{n}^{m}$ constitute a Liouville integrable system. Moreover, $\left\{E_{1}^{m}, W_{r}^{m}\right\}=0$ for any $r$ due to Lemma 3.2. Thus, in order to have $\left\{H_{1}^{m}, W_{r}^{m}\right\}=0$ for any $r$ we have to demand $\left\{V_{1}^{(k)}, W_{r}^{m}\right\}=0$ or, equivalently, $L_{Y_{r}^{m}}\left(V_{1}^{(k)}\right)=0$, for all $r$ which is satisfied only in the mentioned four cases. 
Let us now turn to algebraic properties of the set of functions $h_{r}^{m}$ in (5.1). Since some of these functions can now commute to the constant $\alpha$ we have to extend the set of function $h_{r}^{m}$ by the constant Hamiltonian $h_{0}=\alpha$ in order to turn it into a Poisson algebra.

Proposition 5.2. The functions $h_{0}^{m}=\alpha, h_{1}^{m}, \ldots, h_{n}^{m}$ in $(5.1)$ for $(m, k)=(0, n),(n, n),(1,-1)$ and $(n+1,-1)$ constitute a Poisson algebra with the commutation relations as in (3.16).

Let us note that since the potentials $V_{r}^{(k)}$ in (5.1) do not appear in the first part of Hamiltonian equations (2.4) they do not appear in the corresponding hydrodynamic system (4.10). Nor does the constant Hamiltonian $h_{0}^{m}=\alpha$. Therefore, the hydrodynamic system corresponding to (3.15) and the one corresponding to (5.1) are exactly the same.

Due to Lemma 5.1, we will on what follows only need the potentials $V_{r}^{(n)}$ and $V_{r}^{(-1)}$. They can be calculated from (5.2) and are in $q$-coordinates given by

$$
V_{r}^{(n)}=q_{r}, \quad V_{r}^{(-1)}=\frac{q_{r-1}}{q_{n}}, \quad r=1, \ldots, n .
$$

Let us now perform the Stäckel transform of the Hamiltonians $h_{r}^{m}$ in (5.1) with respect to the parameter $\alpha$. It means that we first solve the relation $h_{1}^{m}=\tilde{\alpha}$, i.e., $E_{1}^{m}+\alpha V_{1}^{(k)}=\tilde{\alpha}$, with respect to $\alpha$ which yields

$$
\tilde{h}_{1}^{m}=\alpha=-\frac{1}{V_{1}^{(k)}} E_{1}^{m}+\tilde{\alpha} \frac{1}{V_{1}^{(k)}}
$$

and then replace $\alpha$ with $\tilde{h}_{1}^{m}$ in all the remaining Hamiltonians $h_{r}^{m}, r=2, \ldots, n$, which yields

$$
\tilde{h}_{r}^{m}=\left.h_{r}^{m}\right|_{\alpha \rightarrow \tilde{h}_{1}^{m}}=E_{r}^{m}+\left(-\frac{1}{V_{1}^{(k)}} E_{1}^{m}+\tilde{\alpha} \frac{1}{V_{1}^{(k)}}\right) V_{r}^{(k)}+W_{r}^{m} .
$$

Thus, the Stäckel transform of (5.1) with respect to $\alpha$ attains the form

$$
\begin{aligned}
& \tilde{h}_{1}^{m}=-\frac{1}{V_{1}^{(k)}} E_{1}^{m}+\tilde{\alpha} \frac{1}{V_{1}^{(k)}}, \\
& \tilde{h}_{r}^{m}=E_{r}^{m}-\frac{V_{r}^{(k)}}{V_{1}^{(k)}} E_{1}^{m}+\tilde{\alpha} \frac{V_{r}^{(k)}}{V_{1}^{(k)}}+W_{r}^{m}=\tilde{H}_{r}^{m}+W_{r}^{m}, \quad r=2, \ldots, n,
\end{aligned}
$$

where

$$
\tilde{H}_{r}^{m}=\tilde{E}_{r}^{m}+\tilde{\alpha} \frac{V_{r}^{(k)}}{V_{1}^{(k)}}, \quad \tilde{E}_{r}^{m}=\frac{1}{2} p^{T} \tilde{K}_{r} \tilde{G}_{m} p .
$$

Due to (5.4) the metric $\tilde{G}_{m}$ in $\tilde{h}_{1}^{m}$ is of the form

$$
\tilde{G}_{m}=-\frac{1}{V_{1}^{(k)}} G_{m}
$$

Since $\tilde{G}_{m}$ is a conformal deformation of the metric $G_{m}$ by $V_{1}^{(k)}$, which in the considered four cases $(m, k)=(0, n),(n, n),(1,-1),(n+1,-1)$ satisfies $L_{Y_{r}^{m}}\left(V_{1}^{(k)}\right)=0$, all $n-1$ vector fields $Y_{r}^{m}$ are in these four cases Killing vectors for the metric $\tilde{G}_{m}$ as well. Thus, the corresponding functions $W_{r}^{m}=p^{T} Y_{r}^{m}$ are constants of motion not only for $H_{1}^{m}$ but also for $\tilde{H}_{1}^{m}$ and therefore the functions $\tilde{H}_{1}^{m}, \ldots, \tilde{H}_{n}^{m}, W_{2}^{m}, \ldots, W_{n}^{m}$ also constitute a maximally superintegrable system. 
Due to the definition of our Stäckel transform (5.5), on the level of separation relations (5.3) the Stäckel transform renders the following substitution:

$$
\alpha \rightarrow \tilde{h}_{1}^{m}, \quad h_{1} \rightarrow \tilde{\alpha}, \quad h_{r}^{m} \rightarrow \tilde{h}_{r}^{m} \quad \text { for } \quad r=2, \ldots, n .
$$

In consequence, the quasi-separation relations for our four cases have the form

$$
\tilde{\alpha} \lambda_{i}^{n-1}+\lambda_{i}^{k} \tilde{h}_{1}^{m}+\sum_{j=2}^{n} \lambda_{i}^{n-j} \tilde{h}_{j}^{m}=\frac{1}{2} \lambda_{i}^{m} \mu_{i}^{2}+\sum_{s=1}^{n} u_{i s}(\lambda) \mu_{s}, \quad i=1, \ldots, n,
$$

with $u_{i k}$ as in (3.21) (compare with (5.3)). However, applying the canonical transformation $\lambda_{i} \rightarrow \lambda_{i}^{-1}, \mu_{i} \rightarrow-\lambda_{i}^{2} \mu_{i}$ to the quasi-separation relations (5.7) with $(m, k)=(1,-1)$ one obtains the quasi-separation relations (3.20) with $m=n+1$ while in the case $(m, k)=(n+1,-1)$ we receive after this transformation the quasi-separation relations (3.20) with $m=1$ so they can not be considered as new systems. Let us thus focus on two remaining cases: $(m, k)=(0, n)$ and $(n, n)$. For both these cases the Killing tensors $\tilde{K}_{r}$ can be expressed through the Killing tensors $K_{r}(3.3)$ as [1]

$$
\tilde{K}_{r}=K_{r+1}-K_{2} K_{r}, \quad r=1, \ldots, n .
$$

Let us now find whether the functions $\tilde{h}_{r}^{m}$ also constitute a Poisson algebra. As it has been demonstrated in [3]

$$
\left\{\tilde{h}_{r}, \tilde{h}_{s}\right\}=\sum_{i, j=1}^{n}\left(A^{-1}\right)_{r i}\left(A^{-1}\right)_{s j}\left\{h_{i}, h_{j}\right\}
$$

where the $n \times n$ matrix $A$ is of the form

$$
A_{i j}=\delta_{i j}, \quad j=2, \ldots, n, \quad A_{i 1}=-\frac{\partial h_{i}}{\partial \alpha}=-V_{i}^{(k)}, \quad i=1, \ldots, n .
$$

So, in general, there is no guarantee that the functions $\tilde{h}_{r}$ constitute an algebra even if $h_{r}$ do.

Proposition 5.3. In the two cases $(m, k)=(0, n)$ and $(n, n)$ the functions $\tilde{h}_{r}^{m}$ constitute a Poisson algebra, with the structure constants obtained from the structure constants of the algebra of $h_{r}^{m}$ given in Proposition 5.2 through the substitution (5.6).

To see this, it is enough to realize that in both cases $(k, m)=(0, n)$ and $(n, n)$ the formula $(5.9)$ reduces to

$$
\left\{\tilde{h}_{r}^{m}, \tilde{h}_{s}^{m}\right\}=\left\{h_{r}^{m}, h_{s}^{m}\right\} \quad \text { for all } r, s,
$$

while the substitution (5.6) in the right-hand side of (5.10) allows for expressing $\left\{h_{r}^{m}, h_{s}^{m}\right\}$ in terms of the Hamiltonians $\tilde{h}_{r}^{m}$.

Example 5.4. For $n=5, m=0$ and $k=n$ the Hamiltonians $h_{r}^{m}$ in (5.1) constitute a Poisson algebra with the following non-zero brackets:

$$
\left\{h_{2}, h_{5}\right\}=3 \alpha, \quad\left\{h_{3}, h_{4}\right\}=\alpha, \quad\left\{h_{3}, h_{5}\right\}=2 h_{1}, \quad\left\{h_{4}, h_{5}\right\}=h_{2},
$$

and after the Stäckel transform (5.5) the corresponding algebra of $\tilde{h}_{1}^{m}$ has due to (5.6) the following non-zero brackets

$$
\left\{\tilde{h}_{2}, \tilde{h}_{5}\right\}=3 \tilde{h}_{1}, \quad\left\{\tilde{h}_{3}, \tilde{h}_{4}\right\}=\tilde{h}_{1}, \quad\left\{\tilde{h}_{3}, \tilde{h}_{5}\right\}=2 \tilde{\alpha}, \quad\left\{\tilde{h}_{4}, \tilde{h}_{5}\right\}=\tilde{h}_{2} .
$$


Our two cases, that is $(m, k)=(0, n)$ or $(n, n)$, lead after the Stäkel transform to two new non-homogeneous hydrodynamic systems through the Theorem 4.1. They have the form

$$
q_{t_{r}}=\tilde{K}_{r} q_{x}+Y_{r} \equiv \tilde{Z}_{r}, \quad r=2, \ldots, n,
$$

with $Y_{r}$ for $m=0$ and $m=n$ the same as before and with $\tilde{K}_{r}$ given by (5.8).

Example 5.5. For $n=4$ the systems (5.11) attain in Viète coordinates $q$ the form

$$
\begin{aligned}
& {\left[\begin{array}{l}
q_{1} \\
q_{2} \\
q_{3} \\
q_{4}
\end{array}\right]_{t_{2}}=\left[\begin{array}{cccc}
q_{2} & -q_{1} & 0 & 0 \\
q_{1} q_{2} & q_{2}-q_{1}^{2} & -q_{1} & 0 \\
q_{1} q_{3} & 0 & q_{2}-q_{1}^{2} & -q_{1} \\
q_{1} q_{4} & 0 & 0 & q_{2}-q_{1}^{2}
\end{array}\right]\left[\begin{array}{l}
q_{1} \\
q_{2} \\
q_{3} \\
q_{4}
\end{array}\right]_{x}+Y_{2}=\tilde{Z}_{2},} \\
& {\left[\begin{array}{l}
q_{1} \\
q_{2} \\
q_{3} \\
q_{4}
\end{array}\right]_{t_{3}}=\left[\begin{array}{cccc}
q_{3} & 0 & -q_{1} & 0 \\
q_{1} q_{3} & q_{3} & -q_{1}^{2} & -q_{1} \\
q_{1} q_{4} & q_{1} q_{3} & q_{3}-q_{1} q_{2} & -q_{1}^{2} \\
0 & q_{1} q_{4} & 0 & q_{3}-q_{1} q_{2}
\end{array}\right]\left[\begin{array}{l}
q_{1} \\
q_{2} \\
q_{3} \\
q_{4}
\end{array}\right]_{x}+Y_{3}=\tilde{Z}_{3},} \\
& {\left[\begin{array}{l}
q_{1} \\
q_{2} \\
q_{3} \\
q_{4}
\end{array}\right]_{t_{4}}=\left[\begin{array}{cccc}
q_{4} & 0 & 0 & -q_{1} \\
q_{1} q_{4} & q_{4} & 0 & -q_{1}^{2} \\
0 & q_{1} q_{4} & q_{4} & -q_{1} q_{2} \\
0 & 0 & q_{1} q_{4} & q_{4}-q_{1} q_{3}
\end{array}\right]\left[\begin{array}{l}
q_{1} \\
q_{2} \\
q_{3} \\
q_{4}
\end{array}\right]_{x}+Y_{4}=\tilde{Z}_{4},}
\end{aligned}
$$

where

$$
\begin{array}{ll}
\text { for } m=0: & Y_{2}=(0,0,0,1)^{T}, \quad Y_{3}=\left(0,0,2, q_{1}\right)^{T}, \quad Y_{4}=\left(0,3,2 q_{1}, q_{2}\right)^{T}, \\
\text { for } m=4: & Y_{2}=\left(0, q_{2}, 2 q_{3}, 3 q_{4}\right)^{T}, \quad Y_{3}=\left(0, q_{3}, 2 q_{4}, 0\right)^{T}, \quad Y_{4}=\left(0, q_{4}, 0,0\right)^{T},
\end{array}
$$

which constitute Lie algebras with the following nonzero elements given by Theorem 4.1 and (3.16)

$$
\begin{array}{ll}
m=0: & {\left[\tilde{Z}_{2}, \tilde{Z}_{4}\right]=-2 \tilde{Z}_{1},} \\
m=4: & {\left[\tilde{Z}_{2}, \tilde{Z}_{3}\right]=\tilde{Z}_{3}, \quad\left[\tilde{Z}_{2}, \tilde{Z}_{4}\right]=2 \tilde{Z}_{4},}
\end{array}
$$

where $\tilde{Z}_{1}=\left(q_{1}, q_{2}, q_{3}, q_{4}\right)_{x}^{T}$.

\section{References}

[1] Błaszak M., Separable systems with quadratic in momenta first integrals, J. Phys. A: Math. Gen. 38 (2005), 1667-1685, nlin.SI/0312025.

[2] Błaszak M., Marciniak K., From Stäckel systems to integrable hierarchies of PDE's: Benenti class of separation relations, J. Math. Phys. 47 (2006), 032904, 26 pages, nlin.SI/0511062.

[3] Błaszak M., Marciniak K., On reciprocal equivalence of Stäckel systems, Stud. Appl. Math. 129 (2012), 26-50, arXiv:1201.0446.

[4] Błaszak M., Marciniak K., Classical and quantum superintegrability of Stäckel systems, SIGMA 13 (2017), 008, 23 pages, arXiv:1608.04546.

[5] Błaszak M., Sergyeyev A., Natural coordinates for a class of Benenti systems, Phys. Lett. A 365 (2007), 28-33, nlin.SI/0604022.

[6] Błaszak M., Sergyeyev A., A coordinate-free construction of conservation laws and reciprocal transformations for a class of integrable hydrodynamic-type systems, Rep. Math. Phys. 64 (2009), 341-354.

[7] Bolsinov A.V., Jovanović B., Noncommutative integrability, moment map and geodesic flows, Ann. Global Anal. Geom. 23 (2003), 305-322, math-ph/0109031. 
[8] Boyer C.P., Kalnins E.G., Miller Jr. W., Stäckel-equivalent integrable Hamiltonian systems, SIAM J. Math. Anal. 17 (1986), 778-797.

[9] Dolan P., Kladouchou A., Card C., On the significance of Killing tensors, Gen. Relativity Gravitation 21 (1989), 427-437.

[10] Ferapontov E.V., Integration of weakly nonlinear hydrodynamic systems in Riemann invariants, Phys. Lett. A 158 (1991), 112-118.

[11] Ferapontov E.V., Fordy A.P., Non-homogeneous systems of hydrodynamic type, related to quadratic Hamiltonians with electromagnetic term, Phys. D 108 (1997), 350-364.

[12] Ferapontov E.V., Fordy A.P., Separable Hamiltonians and integrable systems of hydrodynamic type, J. Geom. Phys. 21 (1997), 169-182.

[13] Ferapontov E.V., Fordy A.P., Commuting quadratic Hamiltonians with velocity dependent potentials, Rep. Math. Phys. 44 (1999), 71-80.

[14] Hietarinta J., Grammaticos B., Dorizzi B., Ramani A., Coupling-constant metamorphosis and duality between integrable Hamiltonian systems, Phys. Rev. Lett. 53 (1984), 1707-1710.

[15] Kalnins E.G., Miller Jr. W., Reid G.J., Separation of variables for complex Riemannian spaces of constant curvature. I. Orthogonal separable coordinates for $\mathrm{S}_{n \mathbf{C}}$ and $\mathrm{E}_{n \mathbf{C}}$, Proc. Roy. Soc. London Ser. A 394 (1984), 183-206.

[16] Marchesiello A., Šnobl L., Winternitz P., Three-dimensional superintegrable systems in a static electromagnetic field, J. Phys. A: Math. Theor. 48 (2015), 395206, 24 pages, arXiv:1507.04632.

[17] Marikhin V.G., On three-dimensional quasi-Stäckel Hamiltonians, J. Phys. A: Math. Theor. 47 (2014), 175201, 6 pages, arXiv:1312.4081.

[18] Marikhin V.G., Sokolov V.V., On quasi-Stäckel Hamiltonians, Russian Math. Surveys 60 (2005), 981-983.

[19] Mishchenko A.S., Fomenko A.T., Generalized Liouville method of integration of Hamiltonian systems, Funct. Anal. Appl. 12 (1978), 113-121.

[20] Rozhdestvenskii B.L., Sidorenko A.D., Impossibility of the "gradient catastrophe" for slightly non-linear systems, USSR Comput. Math. Math. Phys. 7 (1967), 282-287.

[21] Sergyeyev A., Błaszak M., Generalized Stäckel transform and reciprocal transformations for finitedimensional integrable systems, J. Phys. A: Math. Theor. 41 (2008), 105205, 20 pages, arXiv:0706.1473.

[22] Tsarëv S.P., The geometry of Hamiltonian systems of hydrodynamic type. The generalized hodograph method, Math. USSR-Izv. 37 (1991), 397-419. 